\title{
Assessment of Agreement of Wrist Bone Mineral Density with Spine and Hip Bone Mineral Density in Postmenopausal Women

\author{
Madhuchhanda Hazra Mou ${ }^{1}$, Sadia Sultana ${ }_{1}^{2}$, Pupree Mutsuddy ${ }^{2}$, Suraya Sarmin ${ }^{3}$, Naheed Khan ${ }^{4}$ and Anik Biswas 5 \\ 2 National Institute of Nuclear Medicine \& Allied Sciences (NINMAS), Dhaka \\ 3 Institute of Nuclear Medicine \& Allied Sciences (INMAS), Bogura \\ Sheikh Russel Gastroliver Institute \&Hospital, Dhaka \\ 5 Directorate General of Heath Services (DGHS), Mohakhali, Dhaka
}

Correspondence Address: Dr. Madhuchhanda Hazra Mou, Lecturer (Nuclear Medicine), Dhaka Medical College, Dhaka-1000. E-mail: dr.mouhazra1986@gmail.com

\begin{abstract}
Objective: Worldwide, osteoporosis in postmenopausal women is a common public health problem. The measurement of bone mineral density (BMD) of spine and hip using central dual energy X-ray absorptiometry (DXA) is the most standard technique to diagnose osteoporosis. But in many circumstances, measurement of spine or hip BMD is quite difficult to carry out where wrist BMD can be used as an alternative. Moreover, wrist BMD can be done with smaller, cheaper, portable peripheral devices for screening osteoporosis at primary health care level. The objective of the study was to explore the agreement of wrist BMD with spine and hip BMD in postmenopausal women.
\end{abstract}

Patients and Methods: This observational, cross sectional study was carried out at National Institute of Nuclear Medicine \& Allied Sciences (NINMAS), Bangabandhu Sheikh Mujib Medical University (BSMMU) campus, from July 2017 to June 2018. A total of 110 postmenopausal women referred to NINMAS for BMD measurement were enrolled in this study after fulfilling selection criteria. BMD of spine, right and left hip and wrist of enrolled subjects were measured by dual energy $X$-ray absorptiometry (DXA) scan by Stratos DR Bone densitometer.

Results: Agreement analysis done by kappa statistics revealed kappa value of $0.930(p<0.05), 0.782(p<0.05)$ and $0.635(p<0.05)$ between wrist and spine BMD, wrist and right hip BMD and wrist and left hip BMD, respectively. In Bland-Altman analysis, the mean difference of T-score measured by wrist and spine was $-0.007 \pm 1.361$, wrist and right hip was $\mathbf{- 0 . 6 4 5} \pm 1.271$ and wrist and left hip was $\mathbf{- 0 . 4 5 0} \pm 1.259$ with $95 \%$ of differences were found in between -2.674 and 2.660 , 3.136 and 1.846 and $\mathbf{- 2 . 9 1 7}$ and 2.017, respectively. Positive significant Pearson's correlation was observed between wrist BMD and spine BMD ( $\mathrm{r}=0.664 ; p=0.001)$, wrist BMD and right hip BMD ( $\mathrm{r}=0.719$; $p=0.001)$ and wrist BMD and left hip BMD $(\mathrm{r}=0.727 ; p=0.001)$.

Conclusion: This study results showed an excellent kappa agreement between wrist and spine BMD with good kappa agreement of wrist with right and left hip BMD as well as in Bland-Altman analysis, the mean differences of $T$-score between wrist andspine, wrist and right hip and wrist and left hip were small. The biases between the methods were considered not significant suggesting that these sites can be used interchangeably for measurement of BMD. Hence, wrist BMD can be used with regular skeletal sites (spine and hip) as an effective method of diagnosing osteopenia and osteoporosis by DXA where spine or hip BMD is difficult to carry out as well as it can be used for osteoporosis screening at primary health care level by portable peripheral DXA device to initiate early treatment to reduce fracture risks.

Key words: Osteoporosis, Bone Mineral Density (BMD), Central and Peripheral sites, Postmenopausal women.

Bangladesh J. Nucl. Med. Vol. 22 No. 1 January 2019 Doi : https://doi.org/10.3329/bjnm.v22i1.40504

\section{INTRODUCTION}

Osteoporosis in postmenopausal women is a common public health problem worldwide and its incidence increases with age. Postmenopausal women have greater bone loss because of the hormonal changes associated with menopause (1). The spine, hip and wrist are the skeletal sites where the osteoporotic fractures commonly occur (2). Fracture at the wrist is considered as an early warning sign for the presence of postmenopausal osteoporosis (3).

Bone densitometry is the single best approach to predict osteoporotic fracture risk (1). Among many techniques of bone densitometry, dual energy X-ray absorptiometry (DXA) is the gold standard technique (4). The measurement of bone mineral density (BMD) of spine and hip using central dual energy X-ray absorptiometry (DXA) is today's the most standard technique for evaluating the risk of osteoporotic fractures. Wrist can also be used to measure BMD. BMD measured by DXA 
is reported as a comparison to two norms: the expected BMD from a sex and age-matched healthy population (Z-score); or the expected BMD from a sex-matched young adult healthy population (T-score). The difference between the patient's score and the norm is expressed in standard deviations (SDs) (4). T-score is applicable for postmenopausal women and adult men aged 50 years or more. The World Health Organization (WHO) has defined normal bone mass as a T-score $\geq-1$, low bone mass (osteopenia) as a T-score between -1 and -2.5 , osteoporosis as a T-score $\leq-2.5$ and established osteoporosis as a $\mathrm{T}$-score $\leq-2.5$ in the presence of one or more fragility fractures (5).

Both central and peripheral skeletal sites can be measured to assess BMD with DXA equipments which are of two types, central and peripheral device. Central DXA device is an expensive, large unit which measures bone density commonly in the spine and hip are usually available at tertiary hospitals. Peripheral DXA devices are small, portable, low cost devices and measure bone density in the wrist, heel or finger and are suitable for primary health care level (6).

Studies found that estimating wrist BMD not only correlates with other skeletal sites but also gives better detection of bone loss (7-9). Furthermore, in many conditions such as spinal deformity, previous spinal surgery, hip prosthesis or even overweight (weight more than 300 pounds) hip or spine DXA is quite difficult to carry out. In some conditions, the accuracy of the result is dubious due to other underlying disorders such as vertebral compression fractures or osteoarthritis $(6,9-10)$. In addition, osteophyte and aortic calcification cause T-score discordance between spine and hip and give false negative BMD result $(1,7)$. As an alternative, hand DXA (wrist) provides the patient with several advantages, as it offers comfortable sitting position placing their hand on table and the scan is less affected by the amount of soft tissue (9) and also the above mentioned drawbacks of hip and spine DXA can be overcome with hand DXA. Another advantage of wrist BMD is that it can be done with portable peripheral DXA machine which can be set at primary health care level. So, the aim of this study was to explore the agreement of wrist BMD with spine and hip BMD in postmenopausal women.

\section{PATIENTS AND METHODS}

This observational, cross sectional type of study was carried out at National Institute of Nuclear Medicine \& Allied Sciences (NINMAS), Bangabandhu Sheikh Mujib Medical University (BSMMU) campus, from July 2017 to June 2018. The population of the study was postmenopausal women referred to NINMAS for BMD measurement. A total of 110 postmenopausal women referred to NINMAS for BMD measurement were enrolled in this study according to selection criteria. No patient had history of vertebral, hip or wrist (non-dominant hand) fracture, bisphosphonate therapy, hormone replacement therapy, radiological investigation using contrast media and nuclear medicine studies (within 2 weeks), and hip replacement, hip pin or screw, metallic rod or spinal fusion device in the lumbar spine, metallic rod or screw in wrist of non-dominant hand. Prior to commencement of this study, the study was approved by Medical Research Ethics Committee (MREC) of NINMAS. All the study subjects and their attendants were informed about the potential risk and benefit of the procedure and informed written consent was taken from each of them. BMD was measured on enrolled subjects by dual energy X-ray absorptiometry (DXA) scan which was performed by Stratos DR Bone densitometer. BMD was measured at spine (lumbar vertebrae L1-L4), hip (femoral neck) and wrist of nondominant hand. For assessment of spine BMD, the patient was laid supine on the table of the machine with both legs supported on a cushion to flatten the pelvis and lower lumbar spine. To assess the hip BMD, the patient's feet were placed in a brace so that patient's hips rotate inward. For assessment of wrist BMD, patient had to sit on a chair next to the table with the forearm extended and resting on the table. Analysis of data from DXA was computerized and completely automated (software 3D DXA, Medix DR). BMD was determined by $\mathrm{T}$-score against a BMD value. BMD was classified into three 
groups: normal, osteopenia and osteoporosis. All BMD values were analyzed statistically using the Statistical Package for Social Sciences version 22.0 for Windows 7 professional (SPSS Inc., Chicago, Illinois, USA). Kappa agreement analysis and Bland-Altman plot were done for measuring agreement and Pearson's correlation coefficient test was done to assess correlation of wrist BMD with spine and hip BMD, respectively. A $p$ value of 0.05 or less was considered as statistically significant.

\section{RESULTS}

Out of 110 participants, majority (45.5\%) belonged to age group 51-60 years. The mean age, weight, height and body mass index (BMI) were $58.47 \pm 9.0$ years, $57 \pm 12 \mathrm{~kg}$, $144.6 \pm 5.95 \mathrm{~cm}$ and $27.18 \pm 5.39 \mathrm{~kg} / \mathrm{m}_{2}$, respectively. Distribution of osteoporosis and osteopenia in spine, both hips and wrist are shown in Table I. It was found that osteoporosis was more common in wrist and spine while osteopenia was more prevalent in both hips.

Table 1: Distribution of osteoporosis and osteopenia in different sites of the body

\begin{tabular}{lcc}
\hline Site & Osteoporosis & Osteopenia \\
\hline Spine & $48.2 \%$ & $37.3 \%$ \\
Right hip & $29.1 \%$ & $51.8 \%$ \\
Left hip & $34.6 \%$ & $52.7 \%$ \\
Wrist & $54.5 \%$ & $29.1 \%$ \\
\hline
\end{tabular}

Agreement analysis was done by kappa statistics. It was observed that according to spine BMD, 94 subjects had osteopenia/osteoporosis, out of which 92 (97.9\%) subjects had wrist osteopenia/osteoporosis and two (2.1\%) had normal wrist BMD. A total of 16 patients had normal spine BMD. Among them all the subjects had normal wrist BMD. The result of kappa agreement analysis revealed kappa value of 0.930 ( $p$ $<0.05$ ) indicating excellent agreement between wrist BMD and spine BMD (Table 2).

Table 2: Agreement between wrist BMD and spine BMD ( $n=110)(s=$ significant $)$

\begin{tabular}{|c|c|c|c|c|c|c|}
\hline \multirow[t]{3}{*}{ Wrist } & \multicolumn{4}{|c|}{ Spine } & \multirow{2}{*}{\multicolumn{2}{|c|}{$\begin{array}{l}\text { Kappa } P \text { value } \\
\text { value }\end{array}$}} \\
\hline & \multicolumn{2}{|c|}{$(n=94)$} & \multicolumn{2}{|c|}{$\begin{array}{l}\text { Normal } \\
(\mathrm{n}=16)\end{array}$} & & \\
\hline & $\mathbf{n}$ & $\%$ & n & $\%$ & & \\
\hline Osteopenia/Osteoporosis & 92 & 97.9 & 0 & 0.0 & & $\bar{s}$ \\
\hline Normal & 2 & 2.1 & 16 & 100.0 & 0.930 & 0.001 \\
\hline
\end{tabular}

According to right hip BMD, 89 subjects had osteopenia/osteoporosis, out of which 87 (97.8\%) subjects had wrist osteopenia/osteoporosis and two (2.2\%) had normal wrist BMD. Rest 21 subjects had normal right hip BMD. Among them 16 (76.2\%) subjects had normal wrist BMD and five (23.8\%) had wrist osteopenia/osteoporosis. Measures of agreement showed kappa value of 0.782 with $p<0.05$ considered as good agreement (Table 3).

Table 3: Agreement between wrist BMD and right hip BMD (n=110) (s= significant)

\begin{tabular}{lccccrr}
\hline Wrist & \multicolumn{4}{c}{ Right Hip } & & value \\
& \multicolumn{4}{c}{$\begin{array}{c}\text { Osteopenia/0steoporosis } \\
(\mathrm{n}=\mathbf{8 9})\end{array}$} & $\begin{array}{c}\text { Normal } \\
(\mathrm{n}=\mathbf{2 1})\end{array}$ \\
\hline \multirow{2}{*}{ Osteopenia/Osteoporosis } & 87 & 97.8 & $\mathbf{n}$ & $\mathbf{5}$ & 23.8 & \\
Normal & 2 & 2.2 & 16 & 76.2 & 0.782 & 0.001 \\
\hline
\end{tabular}

According to left hip BMD, 96 subjects had osteopenia/osteoporosis, out of which 89 (92.7\%) subjects had wrist osteopenia/osteoporosis and seven (7.3\%) had normal wrist BMD. A total of 14 subjects had normal left hip BMD. Among them 11 (78.6\%) subjects had normal wrist BMD and three (21.4\%)had wrist osteopenia/osteoporosis. The result of kappa agreement analysis revealed kappa value of 0.635 with $p<0.05$ considered as good agreement (Table 4 ).

Table 4: Agreement between wrist BMD and left hip BMD (n=110) (s=significant)

\begin{tabular}{|c|c|c|c|c|c|c|}
\hline \multirow[t]{4}{*}{ Wrist } & & & & & \multirow{4}{*}{$\begin{array}{l}\text { Kappa } \\
\text { value }\end{array}$} & \multirow[t]{4}{*}{$P$ value } \\
\hline & \multicolumn{4}{|c|}{ Left Hip } & & \\
\hline & \multicolumn{2}{|c|}{$\begin{array}{c}\text { Osteopenia/osteoporosis } \\
(\mathrm{n}=96)\end{array}$} & \multicolumn{2}{|c|}{$\begin{array}{c}\text { Normal } \\
(\mathrm{n}=14)\end{array}$} & & \\
\hline & $\mathbf{n}$ & $\%$ & n & $\%$ & & \\
\hline Osteopenia/Osteoporosis & 89 & 92.7 & 3 & 21.4 & 0.635 & $0.001^{\mathrm{s}}$ \\
\hline Normal & 7 & 7.3 & 11 & 78.6 & & \\
\hline
\end{tabular}

Agreement was also assessed by Bland-Altman analysis. In Bland-Altman analysis, the mean difference of $\mathrm{T}$ score measured by wrist and spine was $-0.007 \pm 1.361$ with $95 \%$ of differences were found in between-2.674 and 2.660. The mean difference of T-score measured by wrist and right hip was $-0.645 \pm 1.271$ with $95 \%$ of differences were found in between -3.136 and 1.846 . The mean difference of T-score measured by wrist and left hip was $-0.450 \pm 1.259$ with $95 \%$ of differences were found in between -2.917 and 2.017 (Figure 1,2,3). 


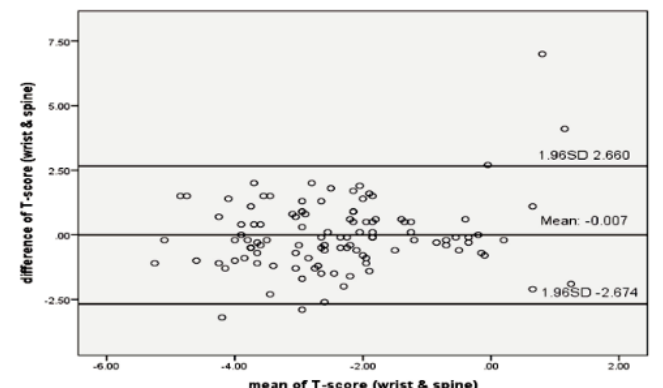

Figure 1: Scatter diagram showing agreement between $\mathrm{T}$-score measured at wrist and spine

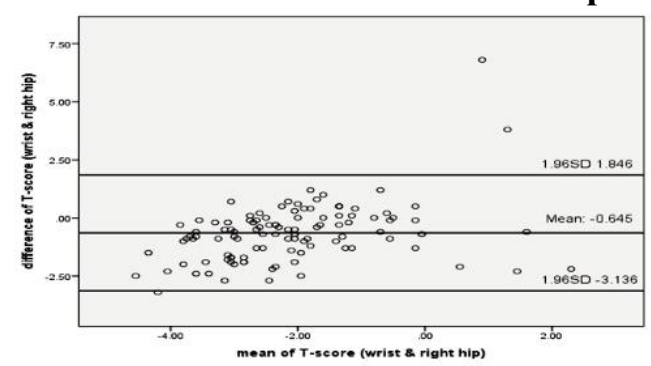

Figure 2: Scatter diagram showing agreement between $\mathrm{T}$-score measured at wrist and right hip

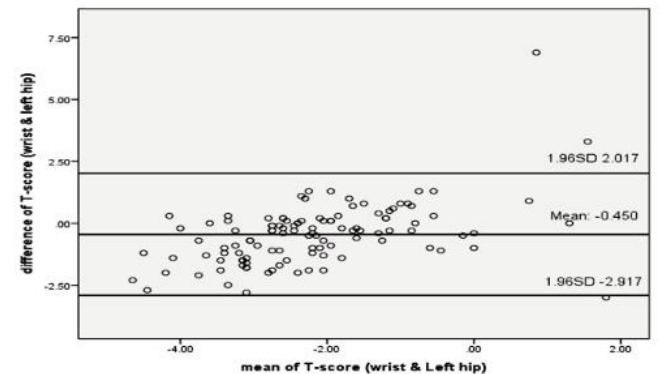

Figure 3: Scatter diagram showing agreement zetween $\mathbf{T}$-score measured at wrist and left hip

Correlation of wrist BMD with spine, right and left hip BMD was measured by Pearson's correlation coefficient test. Positive significant Pearson's correlation was observed between wrist BMD and spine BMD ( $\mathrm{r}=0.664 ; p=0.001)$, wrist BMD and right hip BMD $(\mathrm{r}=0.719 ; p=0.001)$ and wrist BMD and left hip BMD ( $r=0.727 ; p=0.001)$ (Figure 4, 5, 6).

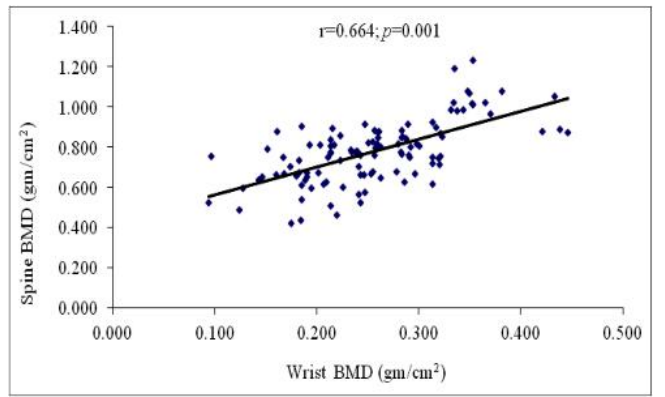

Figure 4: Scatter diagram showing Pearson's correlation between wrist BMD $\left(\mathrm{gm} / \mathrm{cm}_{2}\right)$ and spine BMD $\left(\mathrm{gm} / \mathrm{cm}_{2}\right)$.

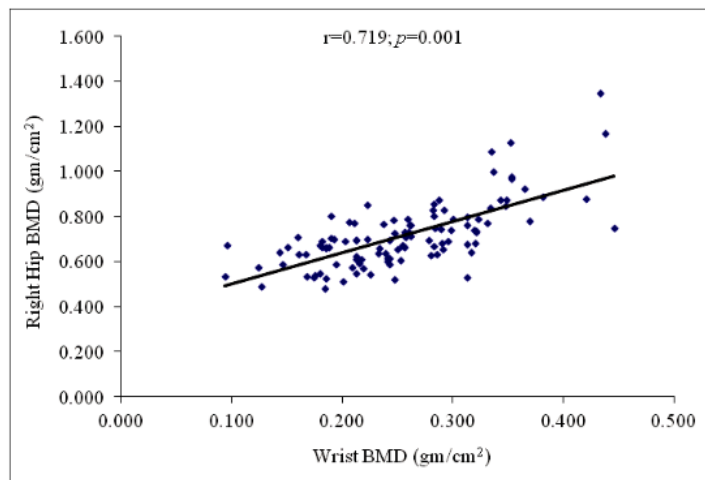

Figure 5: Scatter diagram showing Pearson's correlation between wrist BMD $\left(\mathrm{gm} / \mathrm{cm}_{2}\right)$ and right hip BMD (gm/cm $)$.

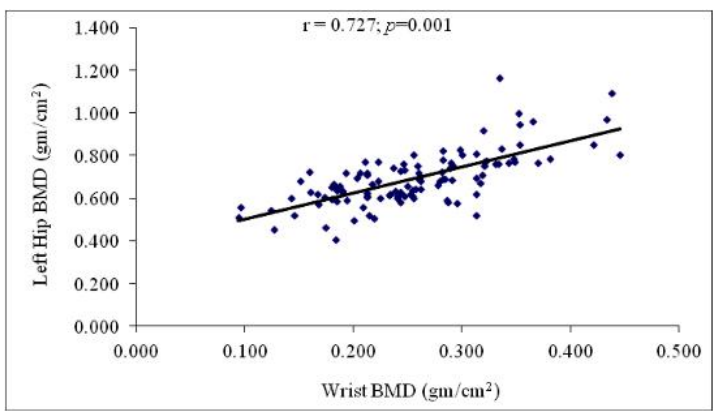

Figure 6: Scatter diagram showing Pearson's correlation between wrist $\mathrm{BMD}\left(\mathrm{gm} / \mathrm{cm}_{2}\right)$ and left hip BMD (gm/ $\left.\mathrm{cm}_{2}\right)$.

\section{DISCUSSION}

In the present study, the mean age was $58.47 \pm 9.0$ years with a range of 43 to 85 years. Eftekhar-Sadat et al.(8) found similar mean age of $57.06 \pm 6.97$ years in their study. On the other hand, Brownbill et al. (9), Leslie et al. (11) and Siris et al. (12) observed higher mean age of 69.1 \pm 9.1 years, $65 \pm 9$ years, and $64.5 \pm 9.3$ years, respectively in their respective studies. In this current study, the mean weight, height and BMI were $57 \pm 12 \mathrm{~kg}, 144.6 \pm 5.95 \mathrm{~cm}$ and27.18 $\pm 5.39 \mathrm{~kg} / \mathrm{m}_{2}$, respectively. Different related studies found similar findings and also variation in weight, height, BMI. Variation in mean age, weight, height and BMI mentioned above may be due to geographical variations, racial, ethnic differences, genetic causes, life style especially their daily food intake and their daily work pattern.

The most important and widely practiced sites to measure BMD are spine and femur (hip), because they are the 
most common sites where osteoporotic fractures take place and cause the maximum impairment of quality of life, morbidity and mortality (5). The spine is more sensitive to the changes associated with treatment therapy (13); this is the reason why spine BMD is a popular method of choice among clinicians to get the optimum BMD measurement. Moreover, Sheu et al. (13) mentioned in their study that hip BMD is also highly acceptable because it is the most predictive site of hip fracture which is clinically the most important fracture. Though spine and hip are most popular sites of BMD measurement, in many circumstances, measuring bone density of the spine and hip region is difficult and sometimes not possible. Therefore, there is a requirement of examining BMD in peripheral skeleton to detect the bone loss and predict the fracture risk. Considering peripheral skeleton such as, measurement of forearm (distal) bone mineral density (BMD) remains valuable to detect fracture risk. In this present study, osteoporosis and osteopenia were found in $48.2 \%$ and $37.3 \%$ subjects in spine, $29.1 \%$ and $51.8 \%$ subjects in right hip, $34.6 \%$ and $52.7 \%$ subjects in left hip, $54.5 \%$ and $29.1 \%$ subjects in wrist indicating that osteoporosis was more common in wrist and spine but osteopenia was more prevalent in hip. Similar type of findings was found in another study by Eftekhar-Sadat et al. (8). They found osteoporosis and osteopenia in $49.5 \%$ and $36.4 \%$ study subjects in lumbar spine, in $24.2 \%$ and $38.4 \%$ study subjects in hip and in $59.6 \%$ and $40.4 \%$ in the wrist BMD measurements. As a result, the investigators found osteoporosis more common in wrist and spine compared to hip whereas osteopenia was more common in hip, which support the present study (8).

In this present study, agreement analysis done by kappa statistics revealed excellent agreement between wrist BMD and spine BMD and good agreement between wrist BMD and right and left hip BMD. In this current study, in Bland-Altman analysis, mean difference of $\mathrm{T}$-score between wrist and spine, wrist and right hip and wrist and left hip were small. The biases between the methods were considered not significant. The differences within mean $\pm 1.96 \mathrm{SD}$ are not clinically important; these methods may be used interchangeably. Kappa agreement analysis and Bland-Altman plot used in is study could not be found in related studies. So, no comparison could be made.

In this current series, Pearson's correlation coefficient test showed a positive significant correlation between wrist and spine BMD ( $\mathrm{r}=0.664 ; p=0.001)$. There was also a positive significant Pearson's correlation between wrist BMD $\left(\mathrm{gm} / \mathrm{cm}_{2}\right)$ and right hip BMD $\left(\mathrm{gm} / \mathrm{cm}_{2}\right)(\mathrm{r}=0.719 ; p=0.001)$ and between wrist BMD $\left(\mathrm{gm} / \mathrm{cm}_{2}\right)$ and left hip BMD $\left(\mathrm{gm} / \mathrm{cm}_{2}\right) \quad(\mathrm{r}=0.727$; $p=0.001$ ). Similarly, Eftekhar-Sadat et al. (8) assessed the role of wrist BMD in diagnosing osteoporosis in postmenopausal women and found a positive correlation between wrist BMD and lumbar BMD $(\mathrm{r}=0.322, p=0.001)$ and between wrist BMD and hip BMD ( $\mathrm{r}=0.468$ and $p<0.001$ ). Brownbill et al. (9) also found that hand BMD has significant correlation with BMD of all other skeletal sites. They concluded that wrist BMD evaluation not only accurately predicts BMD of other skeletal sites but also has a potential in predicting fracture risk due to osteoporosis. Jones et al. (14) comparing distal forearm BMD and BMD at the lumbar spine and femoral neck commented that a large fraction of population at risk of osteoporotic fracture can be identified by forearm BMD with almost $90 \%$ certainty. The correlation coefficient between bone density at the distal (or ultra-distal) forearm and lumbar spine and distal (or ultra-distal) forearm and femoral neck has been reported to be 0.64 and 0.70 , respectively in their study which is comparable to current study (14). Rey and colleagues as cited in Abdel mohsen (7) compared wrist densitometry with hip and lumbar densitometries and observed significant correlation.

This study revealed an excellent agreement of wrist with spine BMD and good agreement with left and right hip BMD as well as wrist BMD has positive 
significant correlation with spine BMD, right and left hip BMD. In Bland-Altman analysis, mean difference of $\mathrm{T}$-score between wrist and spine, right and left hip were small. The biases between the methods were considered not significant. Therefore, wrist BMD similarly as spine and hip BMD can play a precise role in diagnosing osteoporosis in postmenopausal women.

\section{CONCLUSION}

In recent years, the average life expectancy of people is increasing making them more vulnerable of developing osteoporosis which only becomes evident when a fracture occurs. Therefore, early identification of osteoporosis is of prime concern. Study result revealed that osteoporosis was more common in wrist and spine however, osteopenia was more frequent in hip. In this study, wrist BMD showed agreement with spine and hip BMD by both kappa statistics and Bland-Altman analysis. As agreement is found between wrist BMD and spine and hip BMD, wrist can be used with regular skeletal sites (spine and hip) as an effective method of diagnosing osteopenia and osteoporosis where spine BMD or hip BMD is difficult to carry out. Simultaneously, osteoporosis and osteoporotic fractures can be prevented by early detection and treatment through large scale screening of postmenopausal women by wrist BMD measurement with peripheral portable DXA device at primary health care level.

\section{REFERENCES}

1. Brunader R, Shelton DK. Radiologic bone assessment in the evaluation of osteoporosis. American Family Physician 2002;65(7):1357-64. PMID: 11996418

2. Burge R, Dawson-Hughes B, Solomon DH, Wong JB, King A, Tosteson A. Incidence and economic burden of osteoporosis-related fractures in the United States, 2005-2025. Journal of Bone and Mineral Research2007;22(3):465-75. DOI: 10.1359/jbmr.061113
3. Brincat SD, Bincat A, Agius JC. Overview of the pathogenesis and management of postmenopausal osteoporosis. Journal of the Malta College of Pharmacy Practice $2016 \quad ;(22): 14-20$. URI:https://www.um.edu.mt/library/oar//handle/123456789/14276

4. Bonura F. Prevention, screening, and management of osteoporosis: an overview of the current strategies. Postgraduate Medicine 2009;121(4):5-17. https://doi.org/10.3810/pgm.2009.07.2021

5. Fogelman I, Blake GM. Different approaches to bone densitometry. TheJournal of Nuclear Medicine2000;41(12):2015-25. PMID:11138687

6. Radiologyinfo.Bone Density Scan (DEXA).Available from:https://www.radiologyinfo.org/en/info.cfm?pg=dexa[Accessed 6th December 2017].

7. Abdelmohsen AM. Comparison of central and peripheral bone mineral density measurements in postmenopausal women. Journal of Chiropractic Medicine 2017;16(3):199203. https://doi.org/10.1016/j.jcm.2017.08.001

8. Eftekhar-Sadat B, Ghavami M, Toopchizadeh V, Ghahvechi Akbari M. Wrist bone mineral density utility in diagnosing hip osteoporosis in postmenopausal women. Therapeutic Advances in Endocrinology and Metabolism 2016;7(56):207-11. https://doi.org/10.1177/2042018816658164

9. Brownbill RA, Ilich JZ. Validation of the use of the hand for estimating bone mineral density in other skeletal sites by DXA in healthy and osteoarthritic women.Journal of Clinical Densitometry2002;5(3):273-82. DOI: https://doi.org/10.1385/JCD:5:3:273

10. National Osteoporosis Foundation. Bone Density Exam/Testing. Available from: https:/www.nof.org/ patients/diagnosisinformation/bone-density-examtesting/[Accessed 8th January 2018].

11. Leslie WD, Tsang JF, Caetano PA, Lix LM, Manitoba Bone Density Program. Effectiveness of bone density measurement for predicting osteoporotic fractures in clinical practice. The Journal of Clinical Endocrinology \& Metabolism 2007;92(1):77-81. https://doi.org/10.1210/jc.2006-1415

12. Siris ES, Miller PD, Barrett-Connor E, Faulkner KG, Wehren LE, Abbott TA et al. Identification and fracture outcomes of undiagnosed low bone mineral density in postmenopausal women: results from the National Osteoporosis Risk Assessment. JAMA2001;286(22):2815-22. doi: 10.1001/jama.286.22.2815

13. Sheu A, Diamond T. Diagnostic tests:Bone mineral density: testing for osteoporosis. Australian Prescriber 2016;39(2):35-39. doi: 10.18773/austprescr.2016.020

14. Jones T, Davie MW. Bone mineral density at distal forearm can identify patients with osteoporosis at spine or femoral neck. British Journal of Rheumatology 1998;37(5):539-43. https://doi.org/10.1093/rheumatology/37.5.539 